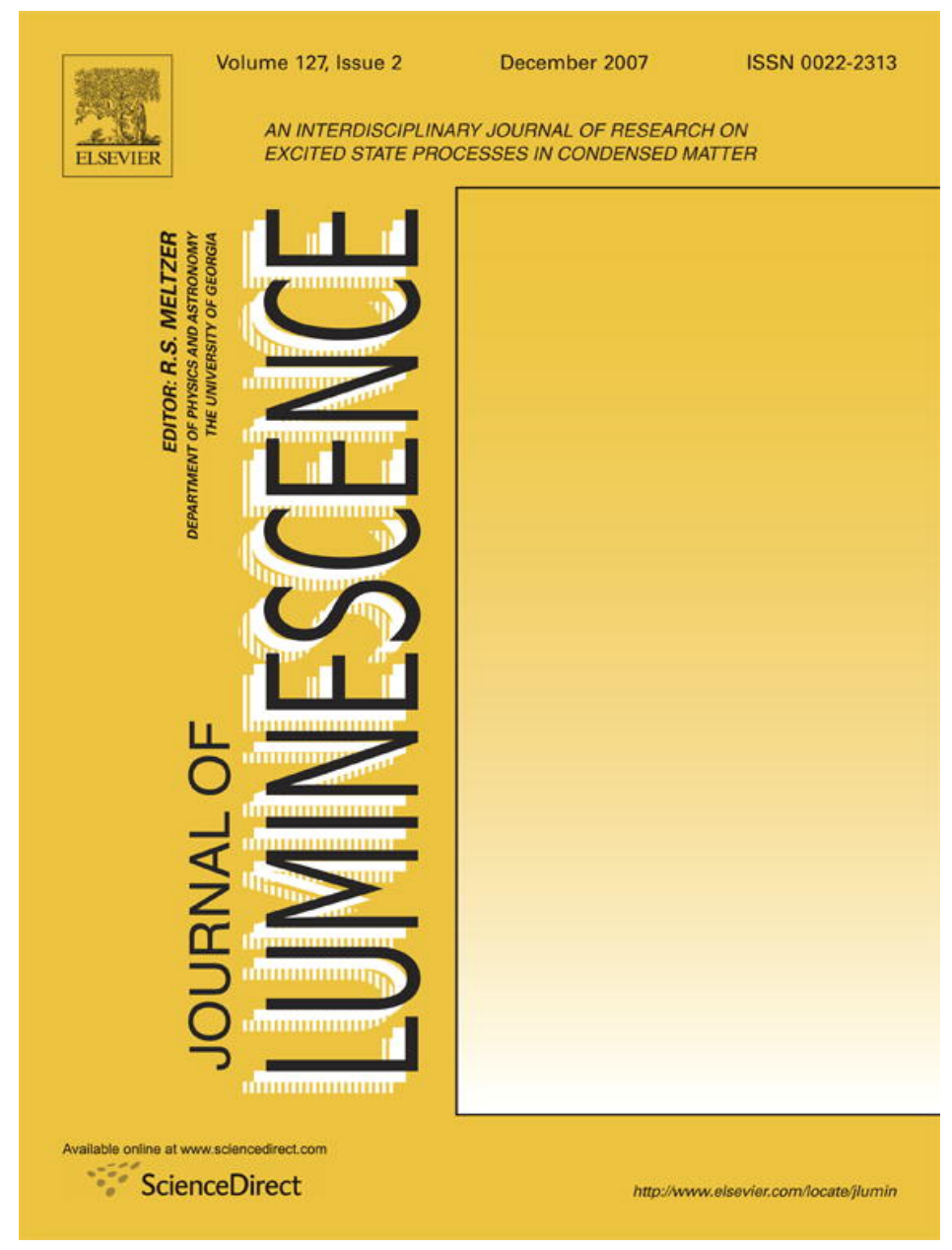

This article was published in an Elsevier journal. The attached copy

is furnished to the author for non-commercial research and education use, including for instruction at the author's institution, sharing with colleagues and providing to institution administration.

Other uses, including reproduction and distribution, or selling or licensing copies, or posting to personal, institutional or third party websites are prohibited.

In most cases authors are permitted to post their version of the article (e.g. in Word or Tex form) to their personal website or institutional repository. Authors requiring further information regarding Elsevier's archiving and manuscript policies are encouraged to visit: 


\title{
Synthesis, morphology and spectroscopy of cubic $\mathrm{Y}_{3} \mathrm{NbO}_{7}: \mathrm{Er}$
}

\author{
Adam Walasek $^{\mathrm{a}}$, Eugeniusz Zych ${ }^{\mathrm{a}, *}$, Jian Zhang ${ }^{\mathrm{b}}$, Shiwei Wang ${ }^{\mathrm{b}}$ \\ ${ }^{a}$ Faculty of Chemistry, University of Wroclaw, 14 Joliot-Curie, 50-383 Wroclaw, Poland \\ ${ }^{\mathrm{b}}$ Shanghai Institute of Ceramics, CAS, 1295 Dingxi Road, Shanghai 200050, PR China
}

Received 11 December 2006; received in revised form 25 February 2007; accepted 28 February 2007

Available online 12 March 2007

\begin{abstract}
Synthesis of $\mathrm{Y}_{3} \mathrm{NbO}_{7}$ :Er powders with the aid of $\mathrm{Li}_{2} \mathrm{SO}_{4}$ flux is reported and spectroscopic properties of the resultant powders are presented. The dopant content varied in the range of $0.1-15$ at $\%$. The materials crystallized in the fluorite-type cubic structure in which all the metal ions - $\mathrm{Y}, \mathrm{Nb}$, and $\mathrm{Er}$ - randomly occupy the same site offered by the host lattice and the O-vacancy is also randomly distributed within the metal surrounding. Transmission electron microscopy images revealed that the agglomeration of particles is very low and the sizes of the grains are around $500 \mathrm{~nm}$. Selected area electron diffraction patterns proved that each grain is monocrystalline. Absorption, excitation, and emission spectra are characterized by relatively broad structures related to the Er ${ }^{3+}$ ion. The broadening results from some inhomogeneity of the activator ion surroundings related to the specific structure of the host lattice. When the Er content is only $0.1 \%$ both photoluminescence and up-converted emission are dominated by a green luminescent band around $550 \mathrm{~nm}$. However, the efficiency of up-conversion is very low. With increasing concentration of the dopant, a red band located around $665 \mathrm{~nm}$ appears and becomes systematically stronger. In up-converted emission, the intensity of the red band surpasses the green one when the Er concentration exceeds 5\%. For low concentrations, the up-conversion occurs through a sequential absorption of two infrared (IR) $(980 \mathrm{~nm})$ photons from the excitation beam by $\mathrm{Er}^{3+}$ ion through excited-state absorption mechanism. For higher concentrations, the energy transfer between two neighboring excited Er ions plays dominant role. Surprisingly, the mechanism of up-converted low-intensity luminescence from ${ }^{2} \mathrm{H}_{11 / 2}$ state seems to diverge from the mechanism characteristic for the ${ }^{4} \mathrm{~S}_{3 / 2}$ level, which conclusion comes from different slopes of the double-log relationships.
\end{abstract}

(C) 2007 Elsevier B.V. All rights reserved.

Keywords: Niobates; Erbium; Luminescence; Up-conversion

\section{Introduction}

$\mathrm{Y}_{3} \mathrm{NbO}_{7}$ crystallizes in a cubic cell with $a=5.25 \AA$, $Z=1[1,2]$. The $\mathrm{Y}_{3} \mathrm{NbO}_{7}$ cubic system may be derived from a fluorite structure of $\mathrm{CaF}_{2}[3,4]$ and possesses very interesting characteristics. First of all the metal ions, $3 \times \mathrm{Y}$ and $1 \times \mathrm{Nb}$, randomly occupy the same site offered by the lattice - the center of a cube. Furthermore, statistically there is one $\mathrm{O}$-vacancy within the eight corners of the cube around the metal site [5], and the vacancy distribution is unsystematic among the eight possible positions in the metal nearest neighborhood. What is more important is that the detailed structural analysis of $\mathrm{Y}_{3} \mathrm{NbO}_{7}$ through

\footnotetext{
*Corresponding author. Tel.: + 48713757248 ; fax: + 48713282348.

E-mail address: zych@wchuwr.pl (E. Zych).
}

X-ray absorption fine structure (EXAFS) measurements showed that the lattice has a tendency to incorporate more than one $\mathrm{O}$-vacancy around the $\mathrm{Y}^{3+}$ ions at the expense of such vacancies in the vicinity of $\mathrm{Nb}^{5+}$ [6]. Clearly, there exists a statistical disorder of the symmetry around the metal ions present in the host lattice. This obviously applies as well to the ions of an activator incorporated into the lattice as a spectroscopically active center.

The described disorder within the nearest environment of the metal sites in the $\mathrm{Y}_{3} \mathrm{NbO}_{7}$ crystal host lattice appeared to us an interesting property, potentially attractive for getting efficient up-converted luminescence (UCL). We expected that the small variations of the positions of the energy levels of individual ions of an activator might facilitate achieving resonant conditions between the activator energy levels involved in the process and the infrared 
(IR) excitation photons. Similar approach was successfully applied by Wells for $\mathrm{CaGdAlO}_{4}$ and $\mathrm{Ca}_{3} \mathrm{Ga}_{2} \mathrm{Ge}_{4} \mathrm{O}_{14}$ doped with $\operatorname{Er}[7,8]$.

Achieving the mentioned resonant or quasi-resonant conditions is a prerequisite to raise an electron to an excited energy state positioned high enough to produce visible luminescence [9] upon absorption of IR photons. This may occur through excited-state absorption (ESA) process or energy-transfer (ET) mechanism [10]. In the former, two (or more) IR photons from the excitation beam are absorbed by the same ion of the activator in turn. In the latter, the ion of activator absorbs one IR photon and the next portion of energy it gets from a neighboring ion, which was also excited within the same time window. The ESA is more probable mechanism of up-conversion in diluted systems, while the ET occurs more readily for concentrated compositions, when necessarily the ions potentially involved in the process are located closer to each other and may interact relatively easily.

\section{Materials and experiments}

The $\mathrm{Y}_{3} \mathrm{NbO}_{7}: x$ at $\%$ Er powders $(x=0.1-15)$ were obtained through a flux-aided route. In a typical synthesis, a stoichiometric mixture of $\mathrm{Nb}_{2} \mathrm{O}_{5}$ and $\mathrm{Y}_{2} \mathrm{O}_{3}$ and $\mathrm{Er}_{2} \mathrm{O}_{3}$ were thoroughly ground in an alumina mortar. The oxides were mixed with $\mathrm{Li}_{2} \mathrm{SO}_{4}$ flux and placed in an alumina crucible. Such a combination was heated in a furnace at $1300{ }^{\circ} \mathrm{C}$ for $5 \mathrm{~h}$. The product was recovered by washing with deionized water and filtering. The powders were dried at $200{ }^{\circ} \mathrm{C}$ for a few hours in air.

The X-ray diffraction (XRD) patterns were measured with IRYS diffractometer using $\mathrm{Cu} K \alpha$ radiation $(\lambda=1.5418 \AA)$ in the range of $2 \theta=10-120^{\circ}$ with the step $\Delta \theta=0.05^{\circ}$. Reflection spectra were measured with UVvis-NIR CARY 500 Scan spectrophotometer with resolution $0.1 \mathrm{~nm}$. Spectra were automatically converted into absorption units by the instrument software. Transmission electron microscopy (TEM) images were taken with JEM2100F (JEOL, Japan) under an operating voltage of $200 \mathrm{kV}$. Photo-excited luminescence (PL) spectra were recorded using Fluorolog-3, Jobin Yvon spectrofluorometer system, which was equipped with a $450 \mathrm{~W}$ Xenon lamp as an excitation source and Hamamatsu R928P photomultiplier as the detector. For UCL spectra measurements, an optical fiber-coupled diode laser tuned to $980 \mathrm{~nm}$ was used as the excitation source, and the UCL spectra were recorded by the same spectrofluorometer as PL emissions. All of the emission spectra were corrected for the spectral response of the measuring system. Experiments were performed at room temperature except one emission spectrum excited with $260 \mathrm{~nm}$ synchrotron radiation, which was taken at $12 \mathrm{~K}$. The resolution of the $12 \mathrm{~K}$ luminescence spectrum was about $0.5 \mathrm{~nm}$. Excitation spectrum of the $\mathrm{Er}$ luminescence down to $100 \mathrm{~nm}$ was also recorded with the use of synchrotron radiation. All spectra using synchrotron radiation were recorded at Superlumi station of HasyLab in Hamburg, Germany.

\section{Results}

\subsection{Structural analysis}

The obtained fine powders were white or pink for lower and higher Er concentrations, respectively. In Fig. 1, we compare the measured XRD patterns for $\mathrm{Y}_{3} \mathrm{NbO}_{7}: 0.5 \% \mathrm{Er}$, $\mathrm{Y}_{3} \mathrm{NbO}_{7}: 15 \% \mathrm{Er}$ and the theoretical one (PDF number 36-1353) for cubic $\mathrm{Y}_{3} \mathrm{NbO}_{7}$. No extra features are observed in the measured spectra compared to the theoretical one. Hence, we can assume that the obtained powders are crystallographically pure.

Electron microscopy additionally proves that the synthesized powders are indeed cubic $\mathrm{Y}_{3} \mathrm{NbO}_{7}$ :Er. In Fig. 2, we show TEM image of the $\mathrm{Y}_{3} \mathrm{NbO}_{7}: 5 \% \mathrm{Er}$ powder together with a representative SAED pattern of a single grain. The results are illustrative for all powders of the various concentrations. In TEM image, we can note that the grains are only loosely agglomerated and the SAED pattern proves that the grains are monocrystalline with cubic structure. The sizes of grains are in the range of $0.5 \mu \mathrm{m}$ and their shapes do not diverge much from spheres in most cases. The relatively small sizes of the grains, as seen in TEMs, attest that the $\mathrm{Li}_{2} \mathrm{SO}_{4}$ flux does not stimulate the particles to a significant growth. On the other hand, the monocrystallinity of the particles attests that the flux allows the atoms making-up the material to form the lattice in an organized manner. Let us yet note that the synthesis performed at $1300{ }^{\circ} \mathrm{C}$ without the aid of the flux does not complete in pure $\mathrm{Y}_{3} \mathrm{NbO}_{7}$. A more detailed analysis and characterization of the flux-aided fabrication procedure of $\mathrm{Y}_{3} \mathrm{NbO}_{7}$ will be published separately.

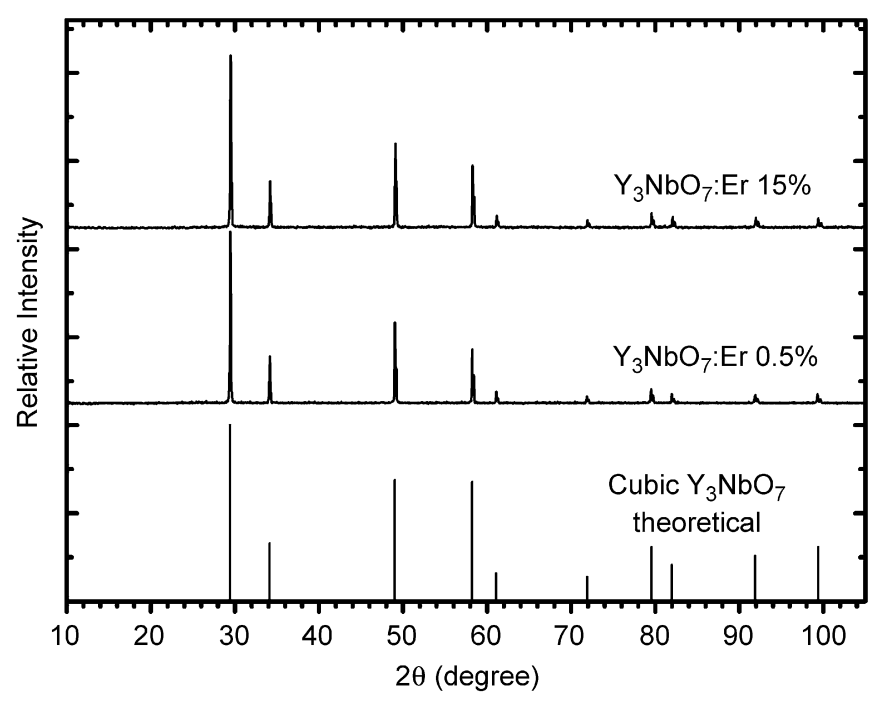

Fig. 1. X-ray diffraction patterns for $\mathrm{Y}_{3} \mathrm{NbO}_{7}$ : Er containing $0.5 \%$ or $15 \%$ of Er and theoretical pattern for cubic $\mathrm{Y}_{3} \mathrm{NbO}_{7}$. 


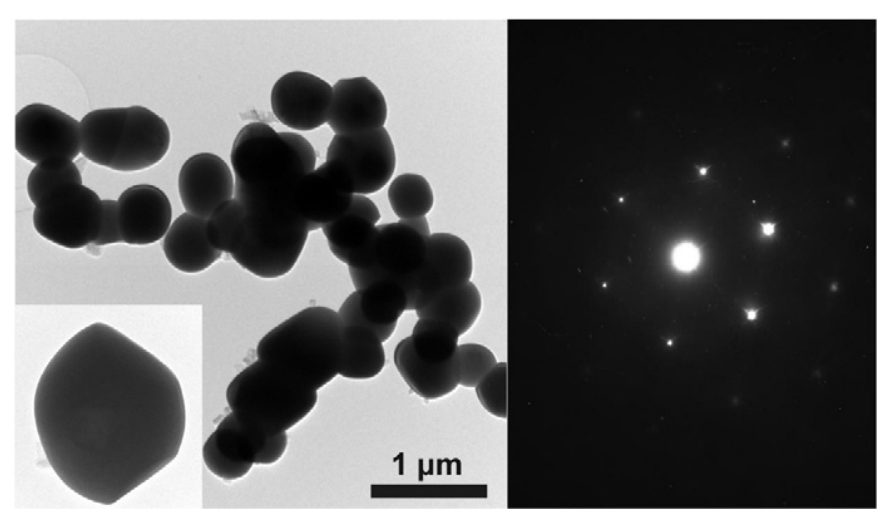

Fig. 2. A representative TEM image of the investigated powders (left) and SAED pattern of a single grain proving the cubic structure of the materials and monocrystalline character of the grains making-up the powders.

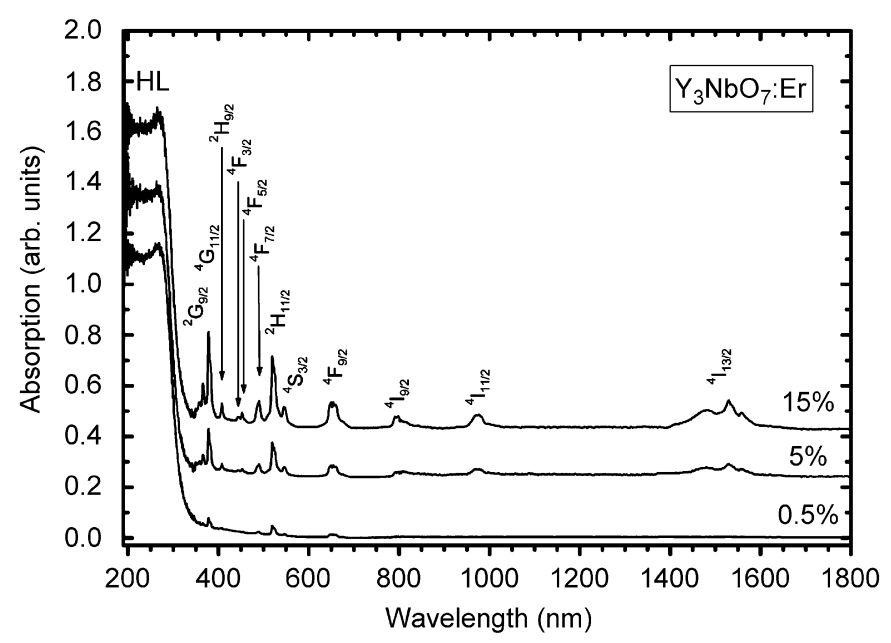

Fig. 3. Absorption spectra (derived from reflectivity) for $\mathrm{Y}_{3} \mathrm{NbO}_{7}: \mathrm{Er}$ powders of different dopant concentrations. Note the saturation of the signal for each sample around $280 \mathrm{~nm}$. This effect indicates that this is the host lattice absorption band. Intensities of the $\mathrm{f}-\mathrm{f}$ transition of $\mathrm{Er}^{3+}$ ion roughly scale with its concentration.

\subsection{Spectroscopic analysis}

In Fig. 3, we show absorption spectra of $\mathrm{Y}_{3} \mathrm{NbO}_{7}: \mathrm{Er}$ powders containing $0.5 \%, 5 \%$ and $15 \%$ of the dopant. All spectra share common characteristics. In the high-energy region, below $300 \mathrm{~nm}$ we find intense bands, whose intensities rise rapidly to saturation around $280 \mathrm{~nm}$ in each spectrum. This also occurs for other Er concentrations, which strongly suggests that the high-energy band results from a fundamental absorption of the $\mathrm{Y}_{3} \mathrm{NbO}_{7}$ host lattice. Such a position of this absorption is quite reasonable for a niobate host. Above $350 \mathrm{~nm}$ we see structures, which are clearly related to the absorption transitions within the $4 \mathrm{f}$ sub-shell of $\mathrm{Er}^{3+}$ ion. Generally, their intensities scale with Er content, although we should be aware that this parameter, cannot be quantitatively judged from measurements of reflectivity. As we supposed above analyzing the crystal structure of $\mathrm{Y}_{3} \mathrm{NbO}_{7}$, the $\mathrm{f}-\mathrm{f}$ transition lines are a

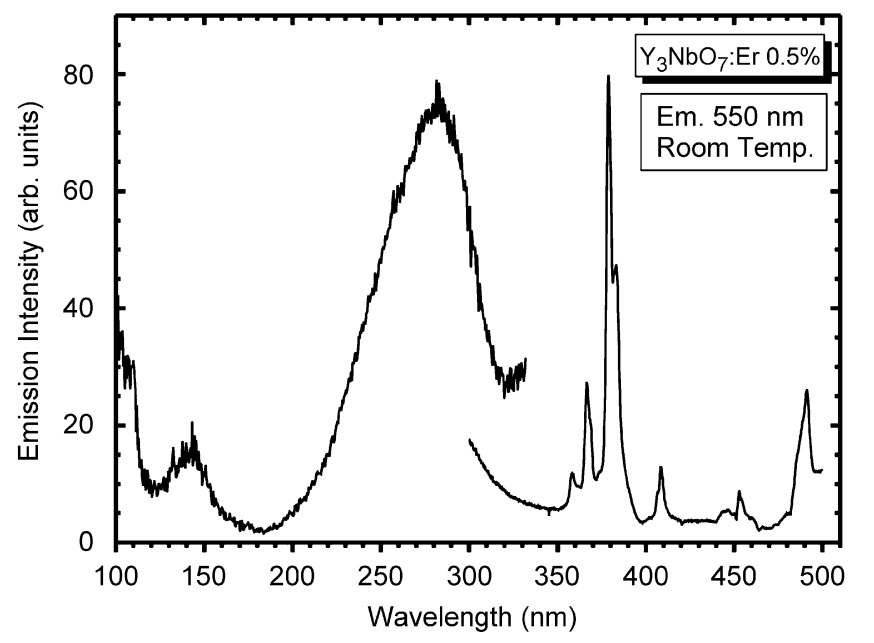

b

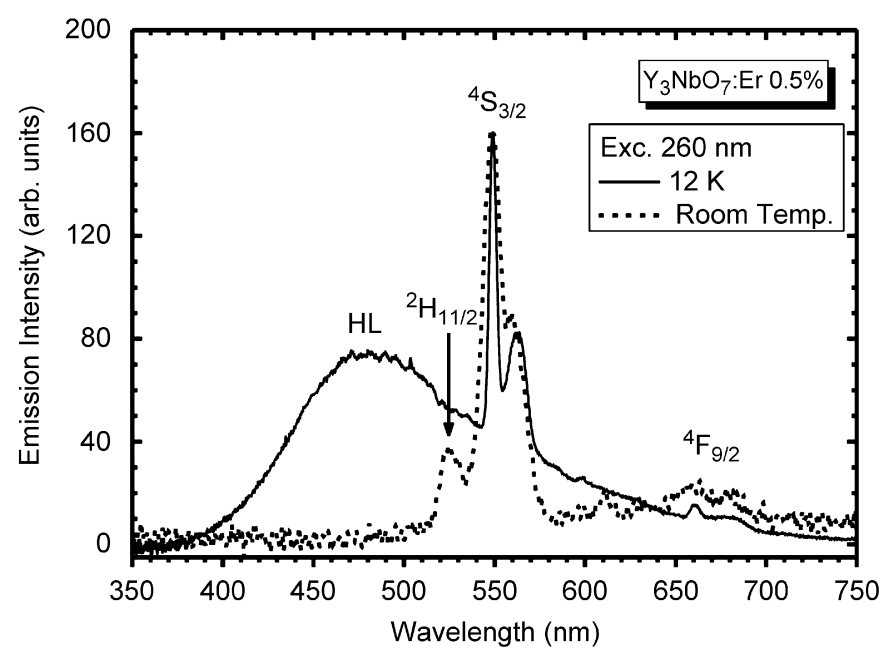

Fig. 4. Room temperature excitation spectrum (a) and both room and $12 \mathrm{~K}$ luminescence spectra of $\mathrm{Y}_{3} \mathrm{NbO}_{7}$ : $\mathrm{Er} 0.5 \%$ powder (b). Note the host lattice luminescence seen only at low temperature and unusually broad for crystalline material the $\mathrm{f}-\mathrm{f}$ emission features even at $12 \mathrm{~K}$.

relatively broad; certainly broader than typically observed in crystals offering a clearly defined symmetry of the activator environment. Hence, we can state that the absorption spectra confirm a structural disorder within the environment of the $\mathrm{Er}^{3+}$ ions incorporated into the $\mathrm{Y}_{3} \mathrm{NbO}_{7}$ host. We shall soon see a similar situation in excitation and emission spectra.

In Fig. 4a, we present a room temperature excitation spectrum of the green $\mathrm{Er}^{3+}$ luminescence located around $550 \mathrm{~nm}$. The lower-energy part of the excitation was measured with regular lab-type spectrofluorometer, while the higher-energy portion was recorded using synchrotron radiation. The excitation spectrum contains a broad band peaking around $280 \mathrm{~nm}$, which coincides with the saturated band in reflection/absorption spectra already presented in Fig. 3. At longer wavelengths, we see structures related to $\mathrm{f} \rightarrow \mathrm{f}$ transitions within $\mathrm{Er}^{3+}$. Since measurements necessarily produce results averaged over a large number of the 
$\mathrm{Er}^{3+}$ ions, we observe the expected inhomogeneous broadening of the recorded structures, somewhat similarly as in glasses.

This conclusion gets perfect support from comparison of the luminescence spectra of the $\mathrm{Y}_{3} \mathrm{NbO}_{7}: 0.5 \% \mathrm{Er}$ powder recorded at room temperature and at $12 \mathrm{~K}$. They are presented in Fig. 4b. As we can see even at temperature of $12 \mathrm{~K}$ the luminescent structures are surprisingly broad. This almost does not change even if resolution of the measurement is further increased (we do not show such a comparison here). The $12 \mathrm{~K}$ emission spectrum contains the expected $\mathrm{f}-\mathrm{f}$ transitions around 550 and $670 \mathrm{~nm}$ superimposed over a very broad band peaking at $490 \mathrm{~nm}$ and covering the $400-700 \mathrm{~nm}$ range of wavelengths. The room temperature emission completely lacks this feature showing that this luminescence is quenched at higher temperatures. Such a broad luminescent structure is usually observed in niobates and tantalates at lower temperatures (sometimes also at ambient conditions, like in $\mathrm{YTaO}_{4}$, an efficient X-ray phosphor [11,12]) and may be generally termed a host-lattice luminescence. Note that at $12 \mathrm{~K}$, the $\mathrm{Er}^{3+}$ emission around $550 \mathrm{~nm}$ results exclusively from the ${ }^{4} \mathrm{~S}_{3 / 2} \rightarrow{ }^{4} \mathrm{I}_{15 / 2}$ radiative relaxation, while its $\mathrm{RT}$ counterpart contains a higher-energy structure resulting from ${ }^{2} \mathrm{H}_{11 / 2} \rightarrow$ ${ }^{4} \mathrm{I}_{15 / 2}$ luminescence. This is a typical situation for $\mathrm{Er}^{3+}$, since the ${ }^{2} \mathrm{H}_{11 / 2}$ state is considered to be populated thermally from the slightly lower energy ${ }^{4} \mathrm{~S}_{3 / 2}$. At still longer wavelengths, we see the red emission resulting from the ${ }^{4} \mathrm{~F}_{9 / 2} \rightarrow{ }^{4} \mathrm{I}_{15 / 2}$ transition. Since even at low temperature the $\mathrm{f}-\mathrm{f}$ transitions of Er produce pretty broad structures, similar to what is seen in glasses, we feel finally convinced that the symmetry of the surroundings of dopant indeed vary to some extent from ion to ion leading to an inhomogeneous broadening of the observed spectral features.

For the systematic analysis of the PL spectra of $\mathrm{Y}_{3} \mathrm{NbO}_{7}$ :Er with different Er content, we chose excitation at $377 \mathrm{~nm}$ instead of $280 \mathrm{~nm}$. The $377 \mathrm{~nm}$ wavelength fits into a relatively intense excitation structure (see Fig. 4a) and excites directly $\mathrm{Er}^{3+}$ ion, which we consider a more reasonable prerequisite for later comparison with UCL spectra than excitation into the host-related band around $280 \mathrm{~nm}$. The $377 \mathrm{~nm}$ light elevates an electron from the ${ }^{4} \mathrm{I}_{15 / 2}$ ground level up to the ${ }^{4} \mathrm{G}_{11 / 2}$ state, from where it may easily fall down to the ${ }^{4} \mathrm{~S}_{3 / 2}$ through non-radiative, multi-phonon relaxation taking advantage of small separation of the crowded electronic states in this region of energies (see Fig. 3 showing absorption spectrum). For UCL we chose $980 \mathrm{~nm}$ stimulation, which fits the excitation of electron from the ground state of $\mathrm{Er}^{3+}$ ion up to the ${ }^{4} \mathrm{I}_{11 / 2}$ level, from which the electron can possibly be elevated further to the ${ }^{4} \mathrm{~F}_{7 / 2}$ upon interception of the energy from another $980 \mathrm{~nm}$ photon $[10,13,14]$. In the following section, we shall see if the $\mathrm{Y}_{3} \mathrm{NbO}_{7}$ :Er system follows our expectations.

Hence, now, we compare the PL spectra excited at $377 \mathrm{~nm}$ and the UCL spectra excited with $980 \mathrm{~nm}$ diode laser for all tested concentrations. They are presented in Fig. 5. In the analyzed visible part of the spectrum, there are generally seen two emission bands located around 545 and $670 \mathrm{~nm}$. The assignment of the observed components is also given in Fig. 5. Note, that the higher-energy band comprises two emissions of different origins. The part of luminescence located at $515-535 \mathrm{~nm}$, whose intensity is relatively low, results from radiative decay of the ${ }^{2} \mathrm{H}_{11 / 2}$ level to the ${ }^{4} \mathrm{I}_{15 / 2}$ ground state. The stronger part of the green emission results from ${ }^{4} \mathrm{~S}_{3 / 2} \rightarrow{ }^{4} \mathrm{I}_{15 / 2}$ transitions.
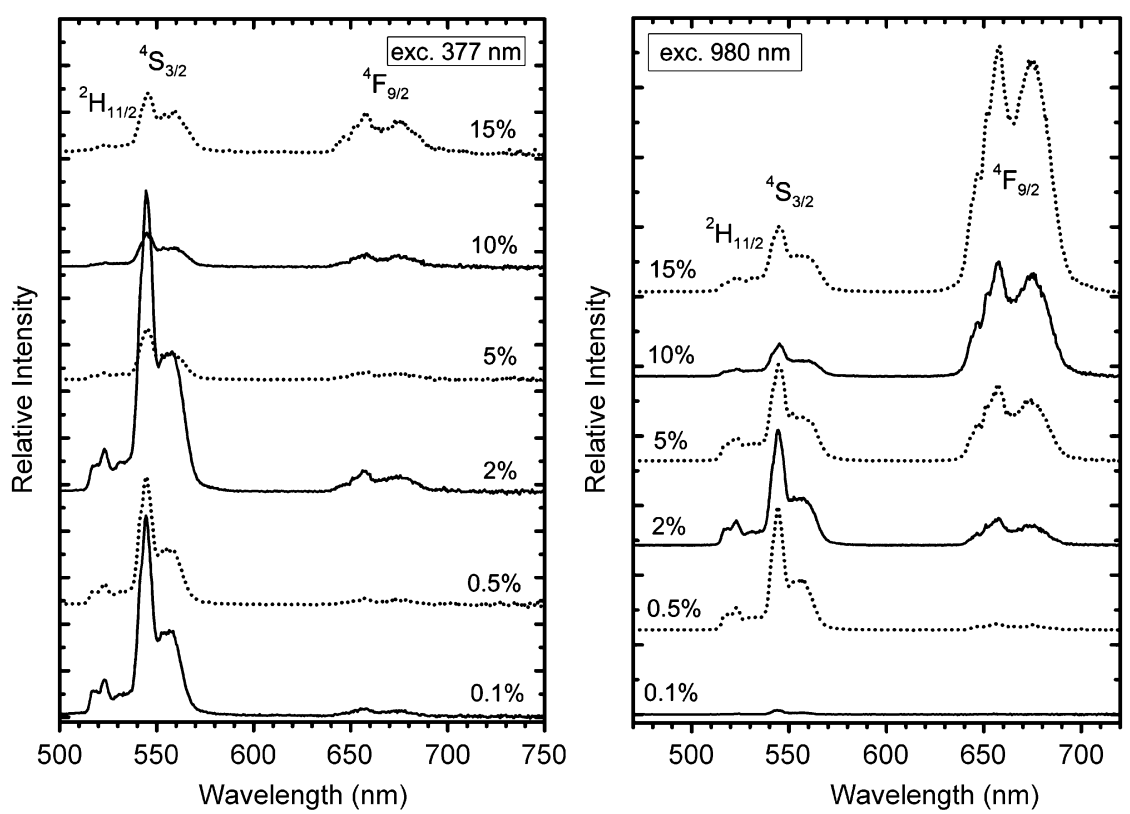

Fig. 5. Room temperature PL (left) and UCL (right) spectra. Note the different Er-concentration dependence of the intensities ratio between the red and green luminescence bands in both types of emissions. 
Finally, the structure around $670 \mathrm{~nm}$ appears due to ${ }^{4} \mathrm{~F}_{9 / 2} \rightarrow{ }^{4} \mathrm{I}_{15 / 2}$ radiative transitions. As expected, all the emission features are much broader than those typically observed in $\mathrm{Er}^{3+}$-doped crystals [15-17]. The existence of inhomogeneous broadening, discussed above, is obvious.

Some characteristics of photoluminescence and upconversion emissions are similar. In both types of spectra, intensity of the red emission band near $670 \mathrm{~nm}$ rises with the increase of the erbium concentration, which means that non-radiative relaxation down to the ${ }^{4} \mathrm{~F}_{9 / 2}$ level becomes more efficient as $\mathrm{Er}$ content increases. Despite some similarities, it is obvious from Fig. 5 that the variations of PL spectra with the dopant concentration are much less profound than the alterations seen in UCL. First of all, we should note that the maximal intensity of PL appears for the sample containing $2 \%$ of Er. Above this concentration the PL efficiency gets reduced, which reflects the appearance of concentration quenching. While we should be very careful concerning exact numbers, qualitatively the situation is clear. In UCL the situation is different. The intensity of the UCL systematically enhances with increasing $\mathrm{Er}$ content within the whole region of investigated concentrations reaching its maximum for the $15 \%$ specimen. Thus, the shorter average distance between Er ions makes the UCL process more effective. As we shall see shortly, this effect has direct connection with the mechanism of UCL.

Another difference easily seen between PL and UCL spectra presented in Fig. 5 is that in PL the intensity of the red emission never surpasses the green one. Thus, in PL the emission color changes from green to yellowish green as the Er content increases from $0.1 \%$ to $15 \%$. We can state that the increase of the Er content from $0.1 \%$ up to $15 \%$ by more than two orders of magnitude - changes only the hue of the color and not the color itself. This is not what we see in UCL. For the lowest concentrations, when the interaction of the Er ions must necessarily be only small, the UCL is purely green, like in PL. However, when the Er content reaches $5 \%$ the color noticeably changes towards yellow and for still higher concentrations of the dopant the UCL color becomes orange, because the band located around $670 \mathrm{~nm}$ takes the major part of the total UCL. Hence, it is quite easy to control the color of the UCL emission by varying the concentration of Er ions. The color may change with increasing Er content from green to yellow and further to orange.

Let us yet comment on the presence of the ${ }^{2} \mathrm{H}_{11 / 2} \rightarrow{ }^{4} \mathrm{I}_{15 / 2}$ luminescence both in PL and UCL. The ${ }^{2} \mathrm{H}_{11 / 2}$ state is located only slightly above the ${ }^{4} \mathrm{~S}_{3 / 2}$ and the ${ }^{2} \mathrm{H}_{11 / 2} \rightarrow{ }^{4} \mathrm{~S}_{3 / 2}$ non-radiative relaxation should be very efficient. This small separation of the two levels allows, however, for the ${ }^{2} \mathrm{H}_{11 / 2}$ state to be easily populated thermally at ambient temperature. That is why the PL spectrum recorded at $12 \mathrm{~K}$ and presented in Fig. 4 does not contain the ${ }^{2} \mathrm{H}_{11 / 2} \rightarrow{ }^{4} \mathrm{I}_{15 / 2}$ luminescence. Hence, at this point it seems quite straightforward that this is indeed the thermal ${ }^{2} \mathrm{H}_{11 / 2} \leftarrow{ }^{4} \mathrm{~S}_{3 / 2}$ backtransfer of energy, which allows for the ${ }^{2} \mathrm{H}_{11 / 2} \rightarrow{ }^{4} \mathrm{I}_{15 / 2}$ radiative transitions to appear. Later on we shall see that the story may be unexpectedly more complex, however, in the case of UCL.

\subsection{Analysis of UCL mechanism and discussion}

To get a closer insight into the possible mechanism of UCL, we investigated the variation of its intensity with irradiation power. In Fig. 6, we show the dependence of $\log I$ on $\log P$ ( $I$ and $P$ stands for emission intensity and laser beam power, respectively) for four selected $\mathrm{Er}$ concentrations, $0.1 \%, 0.5 \%, 5 \%$ and $15 \%$. In all cases the dependences are linear, indicating that the mechanism of UCL does not change with excitation power for fixed $\mathrm{Er}$ content. For the most diluted samples, the lines are practically parallel and their slopes are very close to 2, which value is characteristic for UCL occurring through a two-photon mechanism [10]. For the $0.1 \%$ powder there are only two lines as the red emission is almost totally absent. For the higher concentrations we may note two important changes. First, the slopes for the 545 and $670 \mathrm{~nm}$ emissions drop to about 1 . We shall return to this observation in the next paragraph. Now, let us just state that it is rational to expect that for the higher Er contents the mechanism of the two main UCL emissions changes towards ET. This is reasonable since the reduced separation of $\mathrm{Er}^{3+}$ ions must necessarily facilitate the energy exchange between them. Surprisingly, in the case of the low-intensity UCL from the ${ }^{2} \mathrm{H}_{11 / 2}$ level located around $520 \mathrm{~nm}$ the situation is different - the slope does not drop bellow 1.5 even for highest concentrations of the dopant. This indicates that UCL from the ${ }^{2} \mathrm{H}_{11 / 2}$ state is governed by a more complex mechanism than the UCL from ${ }^{4} \mathrm{~S}_{3 / 2}$ level. This, in turn, suggests that in UCL feeding of the ${ }^{2} \mathrm{H}_{11 / 2}$ level of $\mathrm{Er}^{3+}$ may occur not only through the thermally stimulated ${ }^{2} \mathrm{H}_{11 / 2} \leftarrow{ }^{4} \mathrm{~S}_{3 / 2}$ back-transfer, but also through an additional, alternative pathway.

In Fig. 7, we present how the slope of the $\log I-\log P$ dependence varies for the three UCL emissions within the whole range of the investigated Er concentrations. Clearly, there is a deep similarity between the emissions from ${ }^{4} \mathrm{~S}_{3 / 2}$ and ${ }^{4} \mathrm{~F}_{9 / 2}$ levels. However, with no doubts the situation for the UCL emission from the ${ }^{2} \mathrm{H}_{11 / 2}$ state is different, as we already noted in the above paragraph. As we already mentioned, for the 545 and $670 \mathrm{~nm}$ UCL emissions we can reasonably assume that the mechanism changes from the ESA for the most diluted materials into the one based on ET for powders containing $5-15 \%$ of Er. This is reasonable, since ET may occur only if two ions of the dopant are located close enough in the host lattice to interact and exchange their energy. Because the efficiency of the UCL is the highest for the most concentrated system (see Fig. 5), we can state that the ET mechanism is more favorable for getting an UCL of high efficiency.

The last observation and conclusion appears very useful when it comes to explanation of why the slope of the $\log -\log$ dependence drops to 1 for higher contents of Er. Typically, the slope is understood as giving a direct answer 

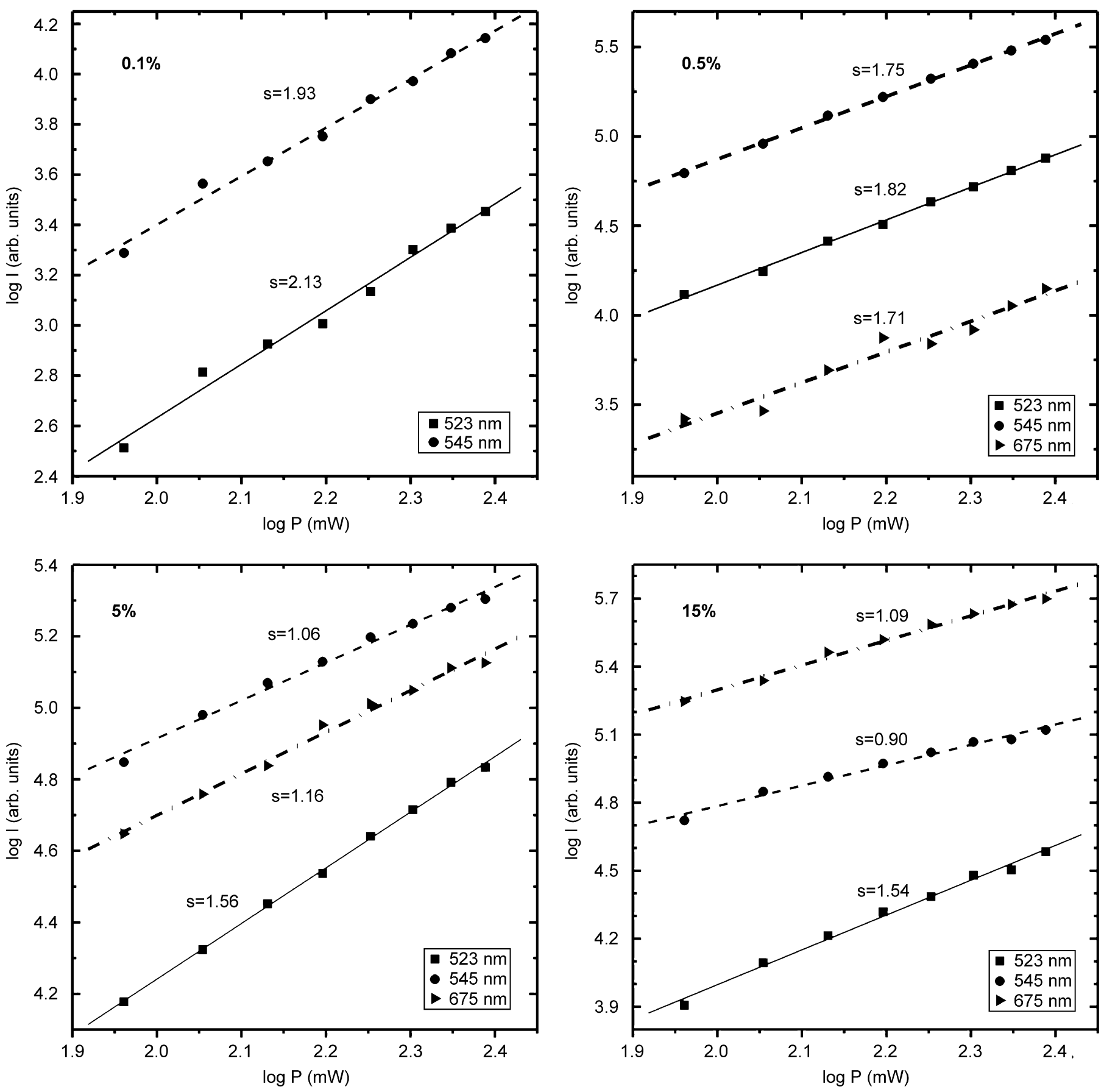

Fig. 6. $\log -\log$ plots for the samples containing $0.1 \%, 0.5 \%, 5 \%$ and $15 \%$ of the dopant. Note variations of the slopes for the $\log I-\log P$ dependences for different transitions as the concentration of Er increases. Only for the UCL from ${ }^{2} \mathrm{H}_{11 / 2}$, the slope does not drop to $\sim 1$ even for the highest concentrations.

about the number of photons from the excitation beam involved into the process of elevation of an electron (independently on the mechanism) into the emitting level. However, it was shown by Pollnau et al. [18] and Dereń et al. $[19,20]$ that such a simple approach is not always valid. Namely, they showed that in systems where the upconverted process of excitation is very efficient compared to non-radiative transitions to the lower levels the $\log I-\log P$ dependence should be close to 1 , exactly as we see for our concentrated compositions. This implies that the $\mathrm{Y}_{3} \mathrm{NbO}_{7}$ :Er phosphor belongs to materials with efficient up-conversion when the dopant content reaches or exceeds $5 \%$. Since we already deduced that for such systems the mechanism of UCL is mostly related to ET we can further conclude that this mechanism is more effective than the ESA one.

The situation is more puzzling for the low-intensity UCL from the ${ }^{2} \mathrm{H}_{11 / 2}$ level around $520 \mathrm{~nm}$. For the reason that the slope of the log-log dependence drops only to the 1.5 we may conclude that in this case the mechanism leading to 


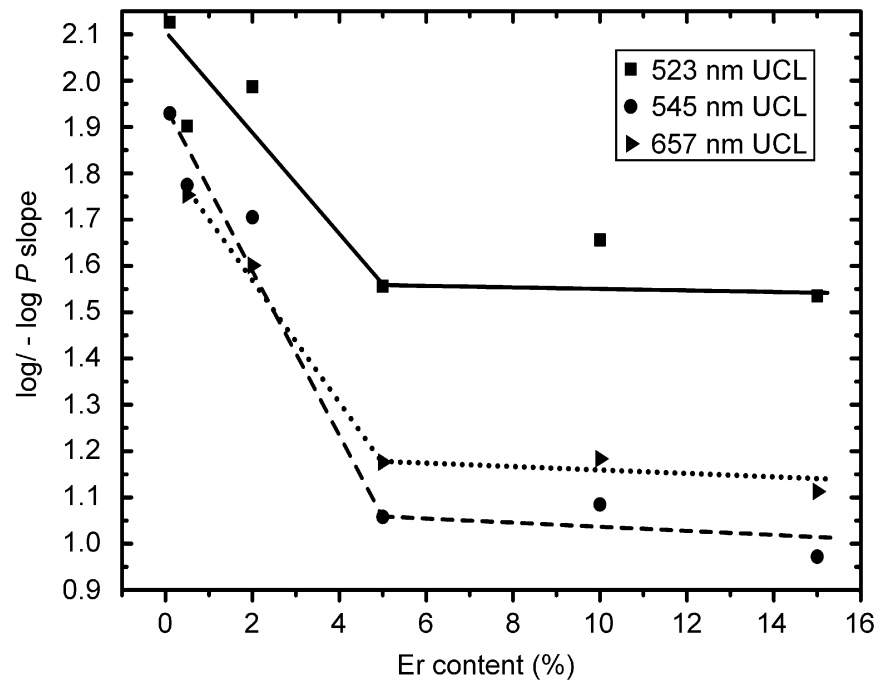

Fig. 7. Variations of the $\log -\log$ slope with Er content within the whole region of investigated concentrations. Note the different dependence of the slope on Er content for the ${ }^{2} \mathrm{H}_{11 / 2}$ UCL emission.

the appearance of the UCL is less efficient than for other two emissions. However, from the findings presented earlier (Figs. 4 and 5) we already postulated that the ${ }^{2} \mathrm{H}_{11 / 2}$ level is populated thermally from the adjacent, slightly lower-lying ${ }^{4} \mathrm{~S}_{3 / 2}$ state. Consequently, both these states should share common characteristics concerning the slope of the log-log dependence. Clearly, what seemed to be very simple at first, appears quite confusing now. It becomes quite apparent that the mechanism of population of the ${ }^{2} \mathrm{H}_{11 / 2}$ state to create the UCL luminescence around $520 \mathrm{~nm}$ may be more complex than usually suggested in literature [21]. Some of our findings question the simple model assuming that only the thermal ${ }^{2} \mathrm{H}_{11 / 2} \leftarrow{ }^{4} \mathrm{~S}_{3 / 2}$ backtransfer of energy stands behind the UCL emission from the ${ }^{2} \mathrm{H}_{11 / 2}$ state. Low-temperature UCL experiments would shed more light on this surprising and confusing observation. At present we do not have any reasonable explanation of this effect. Nevertheless, we wish to share our observation with other researchers as this seems to be rather puzzling phenomenon. $\mathrm{Er}^{3+}$ ion was the first, which was shown to be able to produce visible luminescence upon excitation with IR light of high intensity [22]. Indeed, the localization of electronic levels of $\mathrm{Er}^{3+}$ is favorable for UCL to occur. However, even in this ion, the various processes taking place during the up-conversion are not fully recognized and the literature sometimes omits or at least does not give way to proper attention of some confusing effects.

On the other hand, we cannot totally exclude that the reason of this puzzling effect is very trivial. Namely, that the $520 \mathrm{~nm}$ luminescence results not only from the emission from the ${ }^{2} \mathrm{H}_{11 / 2}$ level of $\mathrm{Er}^{3+}$ ions but also from an unintentional impurity, whose emission appears in the same region of wavelengths. Although the purity of yttria, which seems to be the most probable source of the

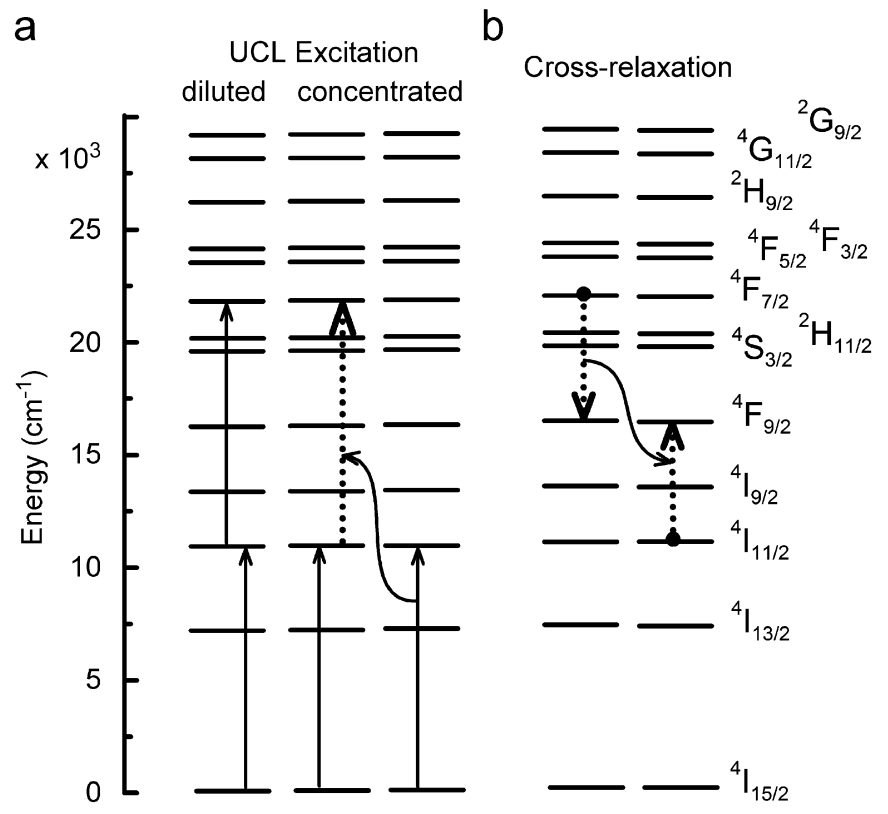

Fig. 8. (a) Schematic depiction of the excitation mechanisms for diluted and concentrated system in UCL. (b) Possible cross-relaxation occurring mainly in concentrated systems leading to significant enhancement of the red luminescence at the expense of the green one especially in the UCL process.

postulated impurity, was $4 \mathrm{~N} 5$, it cannot be ruled out that this is still not enough to avoid the effect we just described.

We wish to say now a few words about the possible reasons of the observed much faster enhancement of the red band intensity in UCL compared to the regular PL with increasing concentration. This fact must mirror variations in relaxation processes from the higher excited states in UCL comparing to PL. The explanation of the differences should thus take into account the fact that the excitations occur through different mechanism in PL and UCL.

Two $980 \mathrm{~nm}$ IR photons may elevate an electron from the ground level up to the ${ }^{4} \mathrm{~F}_{7 / 2}$ state, as shown in Fig. 8a. This may occur through ESA or ET if the concentration of the $\mathrm{Er}^{3+}$ ions is high enough for cooperated effects to occur. In Fig. 8b, we show a possible cross-relaxation process, which may easily explain the stronger enhancement of the red emission in UCL comparing to PL. In the process two $\mathrm{Er}^{3+}$ ions are involved, which makes it more efficient for higher concentrations, in agreement with the observations. The electron elevated up to the ${ }^{4} \mathrm{~F}_{7 / 2}$ state with two IR photons passes part of its energy to a neighboring $\mathrm{Er}^{3+}$ ion having electron excited to the ${ }^{4} \mathrm{I}_{11 / 2}$ level after absorption of one IR photon. As a result we get two ions of $\mathrm{Er}^{3+}$, both excited to the ${ }^{4} \mathrm{~F}_{9 / 2}$ state, from which the red luminescence is created. We postulate that such a cross-relaxation is responsible for the enhancement of the red emission in UCL over the intensity seen in PL. Even more, we can yet note that the slope for the red UCL is about $20 \%$ higher (for $5-15 \%$ of Er) than for the green band (Fig. 7). This slightly higher value, we think, may 
result from the just deduced cross-relaxation process feeding the ${ }^{4} \mathrm{~F}_{9 / 2}$ state responsible for the red luminescence. However, we do not wish to fall into consideration of such tiny effects in this paper, which deal rather with more general phenomena and properties of the title composition.

Obviously, similar cross-relaxation as described above for UCL may also occur in regular PL. However, the $\mathrm{Er}^{3+}$ ions in the state ${ }^{4} \mathrm{I}_{11 / 2}$ necessary for the process to happen are much more numerous in the case of the UCL. In PL, electron may reach this state only due to relaxation from higher levels. Evidently, this may also occur in UCL. However, in the latter yet another, even more efficient channel of getting electron to ${ }^{4} \mathrm{I}_{11 / 2}$ level is present. Namely, $\mathrm{Er}^{3+}$ ions in such states are frequently directly created due to absorption of $980 \mathrm{~nm}$ photons continuously hitting the phosphor. This favors the described crossrelaxation and thus the red part of the emission in UCL gets a significant enhancement comparing to regular PL. This analysis is in agreement with the fact that for the most diluted system both emissions are very similar and almost lack the red part. This occurs since in diluted powders cross-relaxation is very inefficient process for the reason that the average distance between the Er ions is too large.

\section{Conclusions}

Cubic phase powders of $\mathrm{Y}_{3} \mathrm{NbO}_{7}: \mathrm{Er}$ with different doping concentration of $\mathrm{Er}$ ions were synthesized using $\mathrm{Li}_{2} \mathrm{SO}_{4}$ flux as the reaction aid. The powders consist of submicron-sized monocrystalline particles only loosely agglomerated. The absorption, photoluminescence and UCL spectra were investigated. A characteristic feature of all spectra was an inhomogeneous broadening of the $f \rightarrow f$ lines resulted from a structural disorder of the host lattice. By changing the concentration of $\mathrm{Er}^{3+}$ ions, the dominant up-conversion luminescent color of the $\mathrm{Y}_{3} \mathrm{NbO}_{7}$ :Er powders could be turned from green to orange, gradually. The observed enhancement of the red UCL for concentrated systems results from cross-relaxation between two neighboring Er ions, one of which is excited to ${ }^{4} \mathrm{~F}_{7 / 2}$ and the other to ${ }^{4} \mathrm{I}_{11 / 2}$ level. The mechanism of the main UCL emissions changed from ESA for diluted systems towards ET for concentrated powders. However, the weak, highenergy UCL emission from the ${ }^{2} \mathrm{H}_{11 / 2}$ state is probably governed by a more complex, mixed-type mechanism. For concentrated systems, the $\log I-\log P$ plot gives the slope close to 1 , which proves that the up-conversion process is very efficient compared to non-radiative relaxation.

\section{Acknowledgments}

Financial support for this research was supplied by European Community under HasyLab Grant II-05-106 EC, European Social Fund within the "Project of Lower Silesia Research Grants for Increasing Innovations for Ph.D. Students" under Grant Z/2.02/II/2.6/04/04/U/03/05, and by the Natural Science Foundation of China under Grant 50372075.

\section{References}

[1] J.G. Allpress, H.J. Rossell, J. Solid State Chem. 27 (1979) 105.

[2] M. Wakeshima, H. Nishimine, Y. Hinatsu, J. Phys.: Condens. Matter 16 (2004) 4103.

[3] F. Hund, K. Lieck, Z. Anorg. Allg. Chem. 271 (1952) 17.

[4] PDF \#35-816.

[5] Y. Yokogawa, M. Yoshimura, S. Somiya, Solid State Ionics 28-30 (1988) 1250.

[6] T. Tanaka, N. Ishizawa, M. Yoshimura, F. Maruno, H. Oyanagi, J. Solid State Chem. 114 (1995) 79.

[7] M. Yamaga, N. Kodama, T. Yosida, B. Henderson, K. Kindo, J. Phys.: Condens. Matter 9 (1997) 9639.

[8] J.-P.R. Wells, T.P.J. Han, M. Yamaga, N. Kodama, H.G. Gallagher, J. Lumin. 87-89 (2000) 1093.

[9] R. Francini, S. Pietrantoni, M. Zambelli, A. Speghini, M. Bettinelli, J. Alloys Compd. 380 (2004) 34.

[10] F. Auzel, Chem. Rev. 104 (2004) 139.

[11] L.H. Brixner, J. Electrochem. Soc. 111 (1964) 690.

[12] L.H. Brixner, Mater. Chem. Phys. 16 (1987) 253.

[13] A.M. Pires, O.A. Serra, M.R. Davolos, J. Alloys Compd. 374 (2004) 181.

[14] A.M. Pires, O.A. Serra, S. Herr, H.U. Güdel, J. Appl. Phys. 98 (2005) 063529-1.

[15] H. Guo, Y. Li, D. Wang, W. Zhang, M. Yin, L. Lou, S. Xia, J. Alloys Compd. 376 (2004) 23.

[16] D. Wang, M. Yin, S. Xia, V.N. Makhov, N.M. Khaidukov, J.C. Krupa, J. Alloys Compd. 368 (2004) 337.

[17] X. Mateos, R. Solé, J. Gavaldá, M. Aguiló, F. Díaz, J. Massons, J. Lumin. 115 (2005) 131.

[18] M. Pollnau, D.R. Gamelin, S.R. Luthi, H.U. Gudel, M.P. Hehlen, Phys. Rev. B 61 (2000) 3337.

[19] P. Dereń, P. Goldner, O. Guillot-Noël, J. Lumin. 119-120 (2006) 38.

[20] P.J. Dereń, R. Mahiou, J.C. Krupa, J. Alloys Compd. 380 (2004) 357.

[21] J. Zhang, W. Qin, D. Zhao, G. De, J. Zhang, Y. Qang, C. Cao, J. Lumin. 119-120 (2006) 341.

[22] F. Auzel, Proc. IEEE 61 (1973) 758. 